\title{
TUTORÍAS DE PRÁCTICAS: REPRESENTACIONES ACERCA DEL ROL DEL TUTOR Y LAS ESTRATEGIAS PEDAGÓGICAS
}

\author{
INTERNSHIP TUTORIALS: REPRESENTATIONS ABOUT THE ROLE OF THE \\ TUTOR AND THE IMPLEMENTED PEDAGOGICAL STRATEGIES
}

\author{
Andrea Ruffinelli (*) \\ Alejandra Morales \\ Soledad Montoya \\ Claudia Fuenzalida \\ Carla Rodríguez \\ Patricia López \\ Carolina González \\ Facultad de Educación \\ Universidad Alberto Hurtado \\ Chile
}

\section{Resumen}

Este artículo presenta un estudio cualitativo sobre prácticas formativas de tutores de prácticas profesionales en siete carreras de pedagogía de una universidad chilena privada perteneciente al Consejo de Rectores $(\mathrm{CRUCH})$. Se analizan inductivamente las representaciones de tutores y practicantes, respecto al rol de los primeros y las estrategias pedagógicas que implementan, considerando aproximaciones teóricas desde los enfoques directivo y constructivista. Los datos - provenientes de entrevistas tras la observación de un video de su tutoría- evidencian en el rol un tránsito hacia el acompañamiento pedagógico, a diferencia del rol burocrático presente en la literatura. También se develan dificultades para tematizar las estrategias, así como coexistencia y valoración de estrategias directivas y constructivistas. En el discurso de los tutores prevalecen estrategias constructivistas, aunque el reporte de prácticas y sugerencias muestra predominio de estrategias directivas. Las estrategias más valoradas por los tutores son las preguntas, mientras los practicantes demandan incrementar la directividad.

Palabras claves: Formación de docentes; tutoría; personal académico docente; práctica pedagógica; método de enseñanza.

\section{Abstract}

$\left({ }^{*}\right)$ Autor para correspondencia: Andrea Ruffinelli

Universidad Alberto Hurtado, Chie

Facultad de Educación

Av Libertador Bernardo O'Higgins 1869,

Santiago, Región Metropolitana

Correo de contacto: aruffine@uahurtado.cl

(C)2010, Perspectiva Educacional Http://www.perspectivaeducacional.cl

RECIBIDO: 30.08 .2019

ACEPTADO: 10.01 .2020

DOI: 10.4151/07189729-Vol.59-Iss.1-Art.1004
This article shows a qualitative study on formative practices of internship tutors in seven pedagogy programs from the School of Pedagogy of a private Chilean university that belongs to the Council of Deans of Chilean Universities (CRUCH). Representations of tutors and interns about the role of the former and the pedagogic strategies they implement are analyzed in an inductive way, considering theoretical points of view from the constructivist and directive approaches. The data was collected from interviews carried out after watching a video record of their tutoring sessions, and shows a tendency towards educational support instead of the bureaucratic role present in the literature. The results show difficulties in the conceptualization of strategies, and the coexistence of and appreciation for both directive and constructivist strategies. The tutors' discourse shows a prevalence of constructivist strategies, although internship reports and suggestions for improvement indicate a predominance of directive strategies. The strategy most valued by tutors are questions, while interns demand more directions.

Keywords: Teacher training; tutorial; academic teaching staff; pedagogic practice; teaching method. 


\section{El problema y su contexto}

Hoy existe consenso acerca de la centralidad de la formación práctica y, particularmente, de la práctica profesional como espacio de integración de saberes y capacidades pedagógicas y como dispositivo formativo de profesionalización clave para el desarrollo reflexivo (Jones \& Ryan, 2014), dada la oportunidad que ofrece de conexión productiva entre saber teórico y práctico (Gelfuso \& Dennis, 2014).

La literatura releva que el contexto y las características de la relación que se establece entre los formadores y los futuros profesionales en este espacio influyen en la reflexión y construcción del saber pedagógico (Douglas \& Ellis, 2011), develando, por una parte, que el tutor (formador de la universidad que acompaña al practicante) se centra en la crítica al proceso formativo docente y su rol para abordarlo, y por otra, que los hallazgos investigativos respecto de la visión de los practicantes son menos concluyentes: algunos señalan que la tutoría reflexiva influye en el mejoramiento de la práctica al promover puentes entre teoría y práctica, pero predominan las publicaciones en que los estudiantes reconocen al tutor en un rol administrativo más que como guía formativo (Hudson, 2005; Janssen, De Hullu \& Tigelaar, 2008).

Las investigaciones que se han focalizado en la relación tutor-practicante-profesor guía (este último, docente de la escuela que acompaña al practicante en la escuela) constatan la fragilidad de esta tríada. Las discusiones tienden a centrarse en cuestiones anecdóticas, basadas en creencias o experiencias, manteniendo al margen la teoría (Chambers \& Armour, 2011) y estando muy presente el paradigma del profesor efectivo: seguir reglas acerca de cómo organizar la enseñanza, los recursos, actividades, etc. (Hudson, Usak \& Savran-Gencer, 2009). En los practicantes predominaría un discurso poco reflexivo y débil en su fundamentación (Simpson, Hasting \& Hill, 2007), manteniendo el statu quo y optando por mostrar acuerdos (Chambers \& Armour, 2011). Sin embargo, los estudios acerca de la mirada de los tutores y de sus efectos formativos continúan siendo escasos (Geber \& Nyanjom, 2009), y los referidos a las prácticas profesionales apuntan más bien a cuestiones pragmáticas como, por ejemplo, el tiempo requerido para esta formación (Cornejo, 2014).

Desde una perspectiva local y empírica, en la Facultad de Educación en estudio, que imparte siete carreras de pedagogía, la centralidad de la línea de prácticas es fundacional, ligada al desarrollo de capacidades reflexivas en sus talleres homónimos asociados a cada práctica (Talleres de Reflexión). Un análisis interno reciente identificó el problema: un trabajo tutorial fundamentalmente a cargo de profesionales externos, con heterogéneos e idiosincráticos perfiles, roles y prácticas formativas que parecen responder más a un conocimiento experiencial 
individual y menos a fundamentos profesionales de construcción colaborativa (Ruffinelli, 2018). En este marco, se plantea esta investigación orientada a la revisión del espacio tutorial en la perspectiva de la formación de docentes reflexivos, con el objetivo de caracterizar las prácticas tutoriales desde la subjetividad de los actores, a fin de identificar lo que en su experiencia favorece la construcción de conocimiento profesional, permitiendo incorporar estos datos a la mejora continua del proceso formativo.

\section{Antecedentes conceptuales y revisión de la literatura}

\subsection{La formación práctica: tensión directividad/constructivismo}

Uno de los componentes claves de la formación inicial de profesores es el de las prácticas en el medio escolar, orientadas a desarrollar un conjunto de capacidades profesionales susceptibles de aprender solo en dicho espacio, en un ejercicio constante de vinculación teoría-práctica desde la reflexión.

En este sentido, Hirmas (2014) e Hirmas y Cortés (2015) subrayan que al intencionar la relación teoría-práctica se produce una tensión entre un enfoque conductista-aplicacionista (o técnicoinstrumental, directivo) y un enfoque crítico-reflexivo (constructivista). El enfoque directivo se focaliza en la transmisión y reproducción de información y pautas de enseñanza, de modo prescriptivo, fragmentado y descontextualizado, asumiendo que antes de la práctica debe dominarse la teoría. En contraste, el enfoque constructivista asume una mirada compleja, comprensiva, multidimensional y situada del proceso de enseñanza-aprendizaje, entendido como una instancia de reflexión y análisis en el que el saber práctico se construye desde la experiencia y los saberes mediante una reflexión que reconfigura los saberes, generando nuevo conocimiento profesional. Las autoras señalan que existiría predominio: del enfoque directivo anclado en la persistencia de prácticas tradicionales pese a los nuevos discursos (Russell, 2012), de la desarticulación entre las carreras de pedagogía y los centros de práctica, y de la dicotomía teoría-práctica (Hirmas, 2014).

La literatura refiere en este espacio a una "tríada formativa": estudiante en práctica-tutorprofesor guía, aunque tiende a observarse una forma diádica, practicante-tutor (Hirmas, 2014). El contexto, las características de la tutoría y la relación entre la díada o tríada influyen en el desarrollo reflexivo (Bates, Ramírez \& Drits, 2009; Gelfuso \& Dennis, 2014) y en la construcción del saber pedagógico (Douglas \& Ellis, 2011), dado el énfasis de la formación reflexiva en las líneas de prácticas (Gelfuso \& Dennis, 2014; Jones \& Ryan, 2014). 


\subsection{El rol del tutor de práctica}

Existen distintas denominaciones que revelan roles, los que van desde el "fiscalizador", que pone el foco en una dimensión instrumental de la práctica docente, hasta el de "promotor" de la acción reflexiva, centrado en la dimensión relacional (Lawson, Çakmak, Gündüz \& Busher, 2015). Ambrossetti (2010) alude a un acompañante con un grado jerárquico superior que proporciona asesoría en distintos ámbitos, asumiéndose como un guía, consejero, instructor y animador. En línea similar, Correa-Molina (2009) le atribuye funciones de vigilancia, asesoría y orientación, así como de promoción del crecimiento personal (Correa-Molina, 2011).

Rubenstein y Thoron (2013) distinguen dos funciones que coexisten en el rol del tutor: promotor de la mejora mediante orientación y reflexión, y evaluador usando juicios sobre el desempeño. Por su parte, Maes, Colognesi y Van Nieuwenhoven (2018), identifican tres roles: 1) acompañar antes y durante la práctica, mediante retroalimentación y calificación de su desempeño; 2) mediar la relación con el medio escolar, y 3) actuar en calidad de experto para el profesor guía, con quien forma equipo para el acompañamiento.

Desde la perspectiva de los tutores, Sánchez y Jara (2014) indican que ellos asumen el rol de apoyar las exigencias personales, sociales y profesionales de los practicantes. Esto implica contención emocional, desarrollo de miradas analíticas de la realidad educativa y propiciar espacios de colaboración. También se ven a sí mismos, aportando nuevas perspectivas a los estudiantes y favoreciendo la vinculación del conocimiento teórico con la práctica y el desarrollo reflexivo. Desde la perspectiva de los practicantes, el tutor se ocuparía de su desempeño curricular, didáctico y disciplinar, siendo percibidos como un gran apoyo en la toma de decisiones didácticas, la organización de contenidos curriculares, la construcción de instrumentos de evaluación y la entrega de directrices para establecer un clima de aula nutritivo, pero no así en la guía de planificaciones (Romero-Jeldres \& Alcaíno-Opazo, 2014), rol que, desde un punto de vista pedagógico, integraría los anteriores.

Ruffinelli (2018) señala que el rol de los tutores de prácticas parece ser bastante discrecional, lo que dependería del tipo de vinculación que este actor tenga con el programa formativo y con su propia representación acerca de su rol. Este rol oscilaría entre un guía o mediador (puente entre la escuela y los saberes profesionales) y la supervisión burocrática (cumplimiento de planificaciones, clases y normas), evidenciando un continuo entre un rol menos directivo y otro más directivo, en el que predomina una visión de tutor como guía o mediador de la reflexión (bisagra) entre las prácticas en los establecimientos educativos y los saberes profesionales: guía la reflexión acerca de la propia práctica, formula preguntas movilizadoras de saberes, 
retroalimenta fortalezas y debilidades, y acompaña en un rol más bien directivo (Jaeger, 2013), que incorpora elementos de una tutoría menos directiva (Moore-Russo \& Wilsey, 2014).

Respecto al tipo de interacciones en la tutoría, si la discusión es más directiva, los practicantes tienen oportunidad de movilizar creencias y saberes, tendiendo puentes para conectar teoría y práctica y para analizar los contextos pedagógicos (Jaeger, 2013). En cambio, cuando la discusión es menos directiva, los practicantes tienen espacio para construir sus propias representaciones de la enseñanza y para explorar nuevas vías de reflexión (Moore-Russo \& Wilsey, 2014).

En síntesis, se identifican cinco roles del tutor, tres de ellos principales: uno más amplio, el de acompañar al practicante por su paso en la escuela, otro evaluativo, ayudando al practicante a lograr los objetivos y calificando, y otro socioafectivo, de contención de situaciones emocionales asociadas a la práctica; y dos roles secundarios: con menor frecuencia aparece un cuarto rol, pedagógico/didáctico/disciplinar vinculado a interacciones del tutor y del estudiante que facilitarían la comprensión y uso apropiado y en contexto de las capacidades profesionales relacionadas a las distintas disciplinas del currículum escolar. Y finalmente un quinto rol, tal vez integrador de los anteriores, el de vinculador entre la teoría y la práctica, en estrecha relación con el desarrollo reflexivo y profesional.

\subsection{Las estrategias pedagógicas en la tutoría de prácticas}

Respecto de las estrategias pedagógicas que utilizan los formadores para promover el desarrollo reflexivo y con ello capacidades profesionales, algunos autores estiman que la mejor estrategia es "reflexionar" permanentemente (Meirieu, 1998; Perrenoud, 2001). Destaca la interacción verbal organizada en preguntas (Correa-Molina, Chaubet, Collin \& Gervais, 2014; Guerra, 2009; Perrenoud, 2001), que Hattie y Timperley (2007) presentan como un modelo de preguntas dirigidas a procesos metacognitivos del estudiante sobre su propia práctica, así como el análisis de estas (Altet, 1996; Cruickshank, 1987; Perrenoud, 2001) por medio de distintos medios: videos, diarios reflexivos (Perrenoud, 2001), situaciones o incidentes críticos (Altet, 1996), portafolios y bitácoras (Korthagen, Kessels, Koster, Lagerwerf \& Wubbels, 2001). También se utiliza la reflexión en base a la teoría (Cruickshank, 1987; Dewey, 1989; Perrenoud, 2001), el recuerdo estimulado (Korthagen et al., 2001), los grupos de reflexión y escritura o de análisis de casos, aprender sobre metodología de investigación (Perrenoud, 2001) y la estructuración de la reflexión, ofreciendo dimensiones de análisis como andamiaje del proceso (Chung \& Van Es, 2014). 
En la línea de la estructuración planteada por Chung y Van Es (2014), ya en 1995 Butler y Winne presentaban un modelo de retroalimentación y autorregulación de tareas de alta complejidad, sustentado en la capacidad para automonitorear el desempeño usando conocimientos, creencias y percepciones del practicante que requieren de ciertas categorías que pueden ser andamiadas paulatinamente, entregando diferentes pistas o preguntas que centren la atención o pongan en cuestionamiento, activando dichas categorías para automonitorear el desempeño.

Ruffinelli (2018) identifica estrategias que despliegan los tutores en el marco de las prácticas profesionales, observando coexistencia de al menos dos tipos de estrategias pedagógicas: las fundadas en una visión constructivista -orientadas a las estrategias menos directivas de MooreRusso y Wilsey (2014), que permiten explorar nuevas reflexiones-y las basada en una visión más directiva -asociadas a las estrategias directivas de Jaeger (2013) movilizadoras de creencias y saberes para conectar teoría y práctica-. La coexistencia de estas estrategias marcaría un continuo que transita entre estrategias directivas dirigidas a la retroalimentación o estrategia de "juicio/sugerencia" destinadas a comunicar un estándar e informar en qué medida el practicante se aproxima a él, emitiendo un juicio que puede ser acompañado de una sugerencia para alcanzarlo, versus (o complemento) una estrategia orientada a la "autorreflexión guiada" a partir de la que el tutor gatilla o media la actualización de saberes y experiencias del practicante, facilitando la reflexión acerca del propio desempeño y la propuesta de alternativas de mejoramiento.

En las estrategias directivas el rol protagónico es del tutor, quien evalúa lo apropiado o no apropiado y/o sugiere las soluciones. Asume un rol tradicional experto, evaluativo y propositivo: critica el desempeño del estudiante, opina, juzga o hace notar errores, evalúa. Las estrategias más directivas comunican al practicante su visión de la situación y lo que ellos habrían hecho al respecto. Bajo el concepto de "retroalimentación" evalúan, hacen sugerencias u ofrecen alternativas, muestran lo que el practicante "no ve", ayudan a ver más allá, y suelen combinar esta práctica con las preguntas acerca de lo que esta visión les ofrece, junto con recoger la visión del propio practicante sobre la situación.

Finalmente, en este mismo contexto de estrategias más directivas o transmisivas, Bocquillon, Dehon y Derobertmasure (2015) han identificado otras estrategias utilizadas por tutores, como la descripción de la práctica, la formulación de preguntas (principalmente vinculadas a la situación observada), el señalar las dificultades o problemas de la práctica, entre otras.

Las estrategias constructivistas, por su parte, promueven el protagonismo del practicante y el formador asume un rol de guía o mediador, evitando emitir juicios y favoreciendo la autorreflexión del estudiante. La estrategia más frecuente es la "pregunta", tal como habría 
señalado Guerra (2009), promoviendo que el estudiante construya sus propias respuestas en base a sus saberes y experiencia, en oposición a ofrecer las respuestas pertinentes desde la perspectiva del formador. También usan la lectura, orientada a la contrastación con la propia práctica. Otros autores (Gervais \& Correa-Molina, 2004; Puig-Cruells, 2004), mencionan la revisión de bibliografías guiadas y, con especial énfasis, el uso de videos, cuya revisión conjunta contribuiría a reconocer los procesos mentales utilizados durante la realización de las clases e interpretarlos, ayudando a disminuir los aspectos subjetivos de la práctica.

Ambos tipos de estrategias -directivas y constructivistas- parecen alternarse en un mismo formador, aunque las estrategias directivas tenderían a ubicarse al final de una sesión, como corolario del trabajo reflexivo o evaluativo. Resulta particularmente interesante que las estrategias más directivas son reportadas cuando las estrategias de tipo constructivista no dan resultados, cuando faltan bases en los saberes en los practicantes o cuando esto se combina con debilidades en las capacidades básicas de los mismos (Ruffinelli, 2018). En este sentido, sería importante considerar la necesidad de manejar un cuerpo de saberes profesionales que deben estar disponibles para ser utilizados en contexto (Ball \& Forzani, 2011; Concha, Hernández, Del Río, Romo \& Andrade, 2013).

En suma, la literatura describe coexistencia de estrategias pedagógicas directivas y constructivistas. Entre las primeras se encuentran aconsejar, enjuiciar y sugerir, junto a la retroalimentación positiva, y entre las segundas las preguntas generadoras de reflexión y estructuración de la reflexión por dimensiones.

\section{Metodología}

Esta investigación se inscribe en los estudios de la subjetividad del actor, desde la perspectiva de las representaciones sociales entendidas como la capacidad de atribuir sentido a la conducta y comprender la realidad mediante un sistema propio de referencias (Moscovici, 1984), guiando la acción y sirviendo a la justificación de conocimientos y prácticas (Wagner, 1994). El diseño responde a un enfoque cualitativo-descriptivo para responder a la pregunta: ¿Cuáles son las representaciones que tienen tutores y estudiantes acerca de las prácticas formativas en las tutorías de prácticas profesionales de las carreras de pedagogía de esta Facultad de Educación?, enfatizando en la caracterización del rol del tutor, las estrategias pedagógicas utilizadas y, entre ellas, las que se identifican como particularmente favorecedoras del desarrollo profesional.

Los datos fueron construidos desde entrevistas individuales a tutores y estudiantes en práctica, realizadas tras la observación del registro audiovisual de una de sus sesiones de tutoría, y desde entrevistas grupales a estudiantes en práctica. El uso de videos es reconocido en la literatura 
como un soporte de potencial andamiaje intrínseco para el desarrollo reflexivo (Blomberg, Sherin, Renkl, Glogger \& Seidel, 2014; Chung \& Van Es, 2014; Gaudin \& Chaliès, 2012).

Los casos estuvieron constituidos por 10 díadas de estudiantes en práctica preprofesional o profesional y sus 10 tutores, de las siete carreras de pregrado que imparte la Facultad ${ }^{1}$. Se seleccionaron las díadas considerando voluntariedad y representatividad general. Los tutores se seleccionaron teniendo en cuenta una relación estable con la institución. Los practicantes se seleccionaron por rendimiento académico promedio, asegurando representaciones más transversales que individuales ligadas a un aprovechamiento significativamente distinto de la formación.

El análisis se inspiró en los principios de la teoría fundamentada (Strauss \& Corbin, 1998), construyendo las categorías del estudio inductiva y deductivamente mediante codificación abierta, axial y selectiva, y saturando mediante método comparativo constante (Lincoln \& Guba, 1985).

\section{Principales resultados}

\subsection{El rol de los tutores}

En el discurso de tutores y practicantes se identifican cuatro roles del tutor, en orden de frecuencia decreciente y con predominancia de los dos primeros: a) directivo (evaluador/retroalimentador), b) constructivista (promotor de la reflexión), c) pilar emocional y d) gestor administrativo. Estos roles tienden a reportarse encarnados de manera mixta y/o alternada en un mismo tutor y/o sesión.

Los roles más recurrentes, según practicantes y tutores, son los de evaluador o retroalimentador (directivo): "Me felicita por las cosas que ella cree que están bien y me hace más bien comentarios por mejorar en vez de decir 'esto está mal, elimínalo de tu clase'. No es negativo, es más bien como una retroalimentación, como una crítica, no sé, positiva, por decirlo de alguna forma" (Practicante), y el de promotor de la reflexión (constructivista): "El enfoque de este profe es mucho más de reflexión, entonces él lo que más nos potencia es que nosotros tenemos que ser profesores reflexivos. Entonces, que siempre cada uno después que hace su clase debe pensar y ver qué pasó, cómo fue y cuestionarse" (Practicante). En el primer caso, el tutor enjuicia el desempeño, realizando sugerencias e indicaciones; en el segundo, el tutor estimula la reflexión

1 Educación Parvularia, Educación Básica, Educación Diferencial, Pedagogía en Biología y Ciencias Naturales, Pedagogía en Matemáticas, Pedagogía en Inglés y Pedagogía para Profesionales. 
sobre la práctica a partir de la retroalimentación o de preguntas. Con frecuencia los practicantes incluyen aquí el rol de mentor profesional como un orientador, un guía que acompaña al futuro docente en el hacerse profesor.

Tutores y practicantes identifican también el rol de pilar emocional y afectivo para el practicante, promoviendo condiciones de acogida, cercanía afectiva, confianza, bienestar, alerta y contención emocional, así como de horizontalidad para la retroalimentación y la consideración del contexto. Finalmente, algunos practicantes identifican el rol de gestor administrativo, en tanto articulador de la relación entre la universidad y el establecimiento educativo.

\subsection{Estrategias pedagógicas}

Identificar las estrategias pedagógicas de la tutoría resultó una tarea desafiante para tutores y practicantes, evidenciando una débil tematización metodológica de la tutoría. Mencionan algunas estrategias pedagógicas y otras se refieren al dispositivo de tutoría. Se distinguen estrategias pedagógicas directivas y constructivistas alternadamente. Las más recurrentes son las de preguntar (constructivista) y sugerir (directiva). Le siguen estructurar en categorías de análisis (constructivista), modelar (directiva) y describir (directiva).

\subsubsection{Estrategias constructivistas}

a) Preguntar: Tutores y practicantes coinciden en su frecuente presencia orientada a generar reflexión sobre el desempeño: "Ella muchas veces pregunta, no impone una idea, sino me da a mí misma preguntas para que yo reflexione. No es como que 'tú tienes que hacer esto asi', sino que hace preguntas, me hace dudar, siempre me hace dudar para que yo misma me dé cuenta. No es como algo que se imponga a mí" (Practicante). Algunos tutores señalan que podría requerir cierto desarrollo reflexivo de los practicantes, por lo que de no estar presente se precisaría implementar otra estrategia más directiva: "Quería en esa retroalimentación que ella se diera cuenta qué pasó, pero ella, como que al final después de que hablara harto, ella me dice 'bueno, pero es que no sé cómo hacerlo' [entonces le digo] no, sí, mira, está bien estructurada tu clase, hablamos con la profe, te falta manejo de tiempos" (Tutor).

b) Estructurar en categorías de análisis: Ofrecer dimensiones para reflexionar, como los momentos de la clase, didáctica, clima, manejo de aula, etc. 


\subsubsection{Estrategias directivas}

a) Sugerir: Los practicantes mencionan proponer, corregir, señalar lo mejorable, trascendiendo a solo juzgar un desempeño: "Muchas veces me dice que esto debería cambiarlo, no está muy bien, no abordé bien el tema, y me deja una ayuda abajo, 'mira, quizás de esta forma te puede ayudar' o 'te propongo que dejes en claro en tu planificación todas las actividades porque eso le sirve a una persona en caso de que tú te ausentes'” (Practicante).

b) Modelar: El tutor muestra cómo hacer, y la mencionan principalmente practicantes.

c) Describir: A partir de la evocación de situaciones puntuales observadas en la clase, emitiendo juicios y/o sugerencias, lo que es relevado principalmente por tutores.

En síntesis, existe dificultad para tematizar metodológicamente la tutoría y hallazgos para estrategias constructivistas y directivas, prevaleciendo discursivamente las primeras y en frecuencia las segundas.

\subsection{Estrategias pedagógicas favorecedoras de construcción de conocimiento profesional docente}

También les resultó desafiante identificar estrategias favorecedoras de construcción de conocimiento profesional. Se registraron igualmente directivas y constructivistas. Estas últimas tienden a ser referidas con mayor frecuencia, particularmente por los tutores, mientras que los practicantes valoran ambas. Sin embargo, las estrategias directivas tuvieron un nivel de descripción concreta mucho mayor que las estrategias constructivistas.

Las estrategias identificadas por ambos actores como más efectivas son: retroalimentación basada en juicios positivos acerca del desempeño (estrategia directiva): "Destacar constructivamente sus aciertos y que tienen que ver con el enfoque con que él enseña, con el valor que le doy a ciertas decisiones que él tomó y que están basadas en el conocimiento de metodologías, de teorías detrás de las decisiones que toma" (Tutor), y las preguntas (estrategia constructivista), especialmente valoradas por los practicantes.

El tipo de pregunta más valorada por los tutores es la que indaga sobre la relación entre la formación y el desempeño observado, con el objeto de generar un contraste de consistencia entre lo aprendido y lo realizado (vinculación teoría-práctica). Los tutores relevan especialmente esta estrategia desde el valor de aprender a preguntar, por sobre enjuiciar y sugerir. Sin embargo, simultáneamente los practicantes valoran estrategias directivas como la retroalimentación positiva, particularmente si se realiza utilizando una pauta o rúbrica diseñada con ese fin. 
También valoran que explícitamente se les indique lo que se debe hacer, se les señale la vinculación teoría-práctica pertinente, o que se revisen antes las planificaciones de la clase.

\subsubsection{Otras estrategias constructivistas favorecedoras}

a) Estructuración del análisis por dimensiones: "Te dirige la entrevista, te dirige un poco el comentario, ordenando las dimensiones, por cómo pasó por todos los puntos, el ambiente de sala, después la toma de decisiones, el uso de las TIC, después los acuerdos y terminar con una organización de la clase" (Practicante).

b) Descripción, reconstrucción verbal del relato de la experiencia de la clase realizada.

c) Análisis de casos o simulaciones (señaladas solo por practicantes): Mediante diarios reflexivos, siempre y cuando el ejercicio incluya su revisión permanente y no solo su escritura y, por sobre todo, que se analice su evolución.

d) Filmación de clases como insumo para el análisis del propio desempeño y la consideración y legitimación de la perspectiva del practicante durante la tutoría.

\subsubsection{Otra estrategia directiva favorecedora}

a) Modelaje grupal (mencionado solo por tutores): tutor o compañeros pueden modelar un desempeño, acompañado de evaluación formativa permanente.

En suma, se identifican estrategias favorecedoras directivas: retroalimentación basada en juicios positivos acerca del desempeño e indicaciones y vinculación explícita de teoría-práctica por parte del tutor y modelaje, y estrategias constructivistas: preguntas, estructuración por dimensiones, reconstrucción de la clase, análisis de casos, simulaciones, diarios reflexivos y filmación de clases.

Se valoran ambos tipos, propiciando, por un lado, la seguridad del practicante al disponer de referentes del deber ser y, por otro, que se resguarden espacios de desarrollo profesional autónomo basado en la autorreflexión, usando esos saberes profesionales que son permanentemente reforzados/recordados por los tutores.

\subsection{Estrategias identificadas en el dispositivo de tutoría}

Entre las estrategias referidas al dispositivo de tutoría, tutores y practicantes coinciden en la existencia de estrategias relativas a cuidar la sistematicidad de la tutoría. Por ejemplo, un tutor releva la retroalimentación inmediata tras la clase del practicante, así como la realización de dos sesiones, una inmediata y otra posterior; mientras, un practicante releva la necesidad de incrementar la presencia física del tutor en la escuela. Un tutor señala la importancia de cuidar 
en las sesiones el generar empatía y confianza, en tanto un practicante menciona observar horizontalidad en estas sesiones, velando por que el protagonista sea el estudiante.

\subsection{Demandas a las tutorías}

\subsubsection{Demandas de estrategias pedagógicas}

Ambos actores tienden a reforzar la necesidad de incrementar las estrategias directivas, en las que el protagonismo es del tutor, señalando explícitamente lo que se debe hacer, aunque divergen en la forma en que deberían tomar esas intervenciones.

Tutores y practicantes coinciden al sugerir la filmación y análisis de clases como estrategia deseable. Sin embargo, el argumento se vincula a su provisión de evidencia para demostrar desempeños, es decir, para ser utilizadas como medio de contraste directivo.

Los practicantes se inclinan por solicitar más modelaje del tutor, más retroalimentación tanto de juicios positivos como negativos respecto del desempeño, hacer más explícitas e incrementar las sugerencias y considerar siempre la dimensión emocional del practicante.

Los tutores señalan la necesidad de estructurar y planificar mejor la tutoría, en términos de planeación de tiempos, temas, metodologías y focos, asumiendo la necesidad de profesionalizar el rol, considerando espacios para analizar las debilidades de los practicantes (conceptuales y otros saberes profesionales) y para establecer mayor vinculación con la planificación de la clase observada, concretamente, revisándola con anticipación y detalle.

Menciones individuales reciben sugerencias de estrategias relativas a incorporar preguntas (estrategia constructivista), en especial aquellas orientadas a que el practicante fundamente teóricamente, reemplazando en esta acción los juicios del tutor, junto con incrementar el tiempo para que el tutor emita juicios a partir de los cuales el practicante pueda reflexionar. En otras palabras, coexisten sugerencias que asumen visiones más directivas o más constructivistas del rol del tutor.

Otras menciones individuales aluden a incrementar el tiempo de la tutoría destinado a reformular la clase a partir de la revisión de teoría en relación con la práctica, o bien a revisar las planificaciones antes de la tutoría y tenerlas disponibles durante ella, para guiarla desde allí, o a ajustarla según las necesidades particulares del practicante.

En síntesis, se constatan demandas por mayor directividad mediante indicaciones, filmación como contraste y modelaje, más preguntas y hacer un abordaje diferenciado de la tutoría según 
necesidades de los practicantes. Aparece también la necesidad de dotar de estructura la tutoría. Llama la atención la demanda por revisar previamente las planificaciones y utilizarlas en la tutoría, relevando una debilidad en el abordaje de la planificación de la enseñanza en este espacio, y relevando el de la implementación.

\subsubsection{Demandas al dispositivo de la tutoría}

Entre las sugerencias al dispositivo, tutores y practicantes coinciden al demandar un incremento en el tiempo de las tutorías, traducido en mayores oportunidades de aprendizaje para los practicantes, quienes estiman que son breves e insuficientes. En esta ampliación del tiempo los practicantes esperan apoyo, acompañamiento personalizado y continuo, revisión efectiva de sus tareas, y mayor presencia del tutor en la escuela.

Los tutores consideran fundamental profesionalizar la tutoría. Con esto se refieren tanto a la necesidad de formación especializada referente a procesos formativos sobre la tutoría y el desarrollo de la reflexión, como a la necesidad de establecer un trabajo en equipo entre tutores, orientado a compartir experiencias y lograr acuerdos y consistencia respecto del dispositivo, sus instrumentos y las estrategias para generar reflexión. Menciones individuales reciben el asegurar la explicitación y conocimiento de los aprendizajes esperados por parte del tutor, aludiendo particularmente al manejo de indicadores explícitos diferenciadores entre una práctica y otra, que permitan definir y organizar la tutoría. También señalan la importancia de asegurar condiciones materiales y emocionales mínimas, en términos de asegurar la disponibilidad de espacios físicos, tiempos, estrategias e instrumentos apropiados para el desarrollo de las tutorías.

Con respecto a la incorporación de otros actores a esta dimensión del proceso formativo, son los tutores quienes sugieren la incorporación de sí mismos al resto del proceso formativo (docencia de cursos). Los practicantes plantean la conveniencia de incorporar a sus pares, profesores guías y estudiantes de la escuela en el proceso formativo de las prácticas. También destacan la necesidad de que los tutores sean especialistas en el ciclo educativo en que acompañan la práctica, y que estén a cargo del Taller de Reflexión (actividad curricular que acompaña cada práctica), simultánea y articuladamente, en lugar de realizar de forma aislada la práctica, como ocurre con frecuencia.

En suma, se demanda al dispositivo mayor tiempo, profesionalización y especialización, e incorporación de otros actores, como los profesores guías. 


\section{Discusión y conclusiones}

Los hallazgos muestran novedades respecto a algunos elementos que plantea la literatura, así como refrendan y afinan otros aspectos del conocimiento acumulado en este campo. Se confirma la coexistencia de los enfoques directivo y constructivista que describen Hirmas (2014) e Hirmas y Cortés (2015), quienes señalan una prevalencia del primero, mientras que Ruffinelli (2018) destaca el predominio del segundo al indagar en dos programas formativos caracterizados por su formación reflexiva. Este nuevo estudio da cuenta de una predominancia discursiva del segundo enfoque, pero práctica del primero.

\subsection{Sobre el rol del tutor}

Aparecen como principales los roles que identifican Rubenstein y Thoron (2013): a) retroalimentador o evaluador, enjuiciando y sugiriendo desempeños, rol predominantemente identificado por los practicantes y también reconocido ampliamente por los tutores, y b) promotor de la reflexión, que también Douglas y Ellis (2011) identifican como menos frecuente, siendo el más relevado por los tutores y también ampliamente reconocido por los practicantes, así como la combinación de ambos roles. También aparece recurrentemente el rol de contención emocional y promotor de cercanía afectiva del tutor, señalado antes por Sánchez y Jara (2014) y menos presente en la literatura, relevando una dimensión transversal del rol, valorada por ambos actores.

Con menor frecuencia aparece un rol de gestor administrativo, como bisagra entre universidad y establecimiento educativo, rol que podría acercarse al que algunos autores describen como el más recurrente y con una connotación más bien de supervisión burocrática (Hudson, 2005; Janssen et al., 2008; Ruffinelli, 2018). Este hallazgo parece dar cuenta de una suerte de superación, en esta institución formadora, de la función mayormente fiscalizadora del tutor, avanzando hacia la función de promoción de la reflexión en el sentido de lo señalado por Lawson et al. (2015).

La coexistencia y combinación de roles directivos/transmisivos y roles constructivistas, en que bajo la mirada de los practicantes prevalecen (y son frecuentemente demandados) los primeros, mientras que desde la perspectiva de los tutores predominan los segundos, parece dar cuenta de unas prácticas docentes históricamente tradicionales y directivas en las que fueron socializados practicantes y formadores durante su vida escolar y que muy probablemente prevalecieron también en la formación docente de ambos actores. Dicha situación podría a su vez explicar la incorporación más bien discursiva del enfoque constructivista, como un deber ser 
pedagógico en que este mismo acto demoniza las aproximaciones más transmisivas (directivas) de enseñanza, aunque persistan en la práctica.

Este hallazgo parece también advertir de una tensión no resuelta entre los formadores acerca de la legitimidad de utilizar estrategias de distinta naturaleza, según los objetivos pedagógicos que se persigan y el contexto de la situación de aprendizaje. Moore-Russo y Wilsey (2014) señalaban que las estrategias constructivistas permiten ampliar el repertorio reflexivo, mientras las estrategias más directivas movilizan creencias y saberes para conectar teoría y práctica (Jaeger, 2013). Es decir, se orientan a fines diferentes, todos ellos necesarios para el desarrollo profesional de los practicantes.

\subsection{Sobre las estrategias pedagógicas}

Los hallazgos refrendan que la estrategia más frecuente es la pregunta (estrategia constructivista), orientada a generar reflexión, tal como han señalado otros autores (Bocquillon et al., 2015; Correa-Molinaet al., 2014; Guerra, 2009; Perrenoud, 2001; Ruffinelli, 2018). La siguen dos estrategias directivas menos descritas con este fin en la literatura especializada: juicio/sugerencia (Ruffinelli, 2018), que aquí asume la forma de aconsejar, incluyendo sugerir, proponer, corregir, señalar lo mejorable y describir enjuiciando (positiva o negativamente) y/o sugiriendo. Luego aparece una estrategia constructivista también descrita en la literatura: estructurar la reflexión ofreciendo categorías de análisis (Chung \& Van Es, 2014) y, finalmente, dos estrategias directivas: modelar o ejemplificar (mostrar o decir lo que se debe hacer) y describir (sin enjuiciar) el desempeño para estimular también la reflexión en torno a hechos, estrategia que antes habían identificado también Bocquillon et al. (2015).

Se ratifica también el hallazgo de Ruffinelli (2018) relativo a que las estrategias directivas tienden a ser una alternativa cuando las estrategias constructivistas no dan resultado, a causa de debilidades en los saberes profesionales y/o capacidades reflexivas de los practicantes. Aquí cabría una hipótesis adicional acerca de una eventual debilidad del tutor para hacer uso pertinente, contextualizado y provechoso de estrategias constructivistas, legitimando la necesidad de poner un foco en la profesionalización de este rol.

\subsection{Estrategias pedagógicas favorecedoras de construcción de conocimiento profesional docente}

Los tutores atribuyen más valor formativo a las estrategias constructivistas, mientras que los practicantes aprecian tanto estrategias directivas como constructivistas. Las más valoradas son las preguntas (estrategia constructivista) y la retroalimentación basada en juicios positivos acerca 
del desempeño. Las preguntas a las que los tutores atribuyen mayor valor formativo son las que permiten confrontar lo aprendido en la universidad con el desempeño observado en la escuela (preguntas de vinculación teoría-práctica), y son relevadas como una estrategia con potencial formativo superior respecto de enjuiciar y sugerir, posibilitando un aprendizaje mucho más profundo.

Los practicantes valoran (y demandan) recibir indicaciones, que se les indique explícitamente qué hacer frente a una situación determinada, evidenciando la persistencia de una visión escolarizada tradicional, mecánica y transmisiva, distante del ejercicio docente profesional autónomo y contextualizado. Por su parte, los tutores tienden a privilegiar (al menos discursivamente) un enfoque más constructivista, estableciendo mediante la reflexión desde las preguntas un puente entre los saberes, la experiencia y la generación de conocimiento profesional, aunque en la práctica, reconocen implementar con frecuencia estrategias más bien directivas, ya sea argumentando su utilidad para de todos modos generar reflexión y aprendizaje o argumentando que ante las debilidades de conocimientos profesionales o capacidades reflexivas de los practicantes aparece como la única opción posible.

La estructuración de la reflexión por dimensiones, en la línea del modelo de Butler y Winne (1995), es otra estrategia favorecedora del desarrollo profesional que valoran tanto tutores como practicantes, y es precisamente en ella que aparece una estrategia constructivista, andamiante, que podría permitir implementar un lugar intermedio entre la directividad que parecerían precisar los practicantes menos aventajados y la construcción de conocimiento profesional más profundo. También, particularmente los tutores valoran la descripción de la clase realizada, el reconstruir los hechos para propiciar la reflexión, el modelamiento (mostrar cómo se hace) y la evaluación formativa, aludiendo al enjuiciamiento del desempeño.

Practicantes y tutores valoran otras estrategias que han experimentado y que la literatura describe para el desarrollo reflexivo, como el análisis de casos, simulaciones, diarios reflexivos y filmación de clases y análisis, enfatizando los practicantes su condición de socialización, es decir, que constituyan insumo de discusión con el tutor y/o pares, y no solo una herramienta de trabajo individual. Entre estas estrategias se valora la filmación y análisis de clases, y pese a que para un grupo de tutores y practicantes su valor radica en la evidencia que provee para demostrar errores, volviéndose así una estrategia más bien controversial, la retroalimentación negativa, también es demandada por los practicantes, quienes exigen más juicios, retroalimentación positiva, modelaje, sugerencias y el que consideren su propia perspectiva, planteando la importancia de su rol activo en una relación de mayor horizontalidad y bidireccionalidad, en línea opuesta a los hallazgos de Chambers y Armour (2011) cuando señalan que los estudiantes tienden a mantener el statu quo, y mostrar acuerdo con el tutor. 
Con todo, la presencia más discursiva que práctica de estrategias constructivistas y la persistencia de la preferencia por estrategias más directivas da cuenta, tal como ya se señalara, de la herencia de unas prácticas docentes tradicionales de la escuela hacia la formación inicial docente. No obstante, parece preciso superar la dicotomía estrategias directivas/transmisivas versus estrategias constructivistas, con miras a una acción pedagógica profesional capaz de determinar, en situaciones educativas, la estrategia más apropiada a los objetivos y el contexto, seleccionada desde un amplio repertorio.

La Tabla 1 resume las estrategias más valoradas y/o usadas por los actores del estudio, estableciendo un paralelo con hallazgos evidenciados previamente en la literatura.

Tabla 1

Estrategias utilizadas

\begin{tabular}{lcl}
\hline $\begin{array}{l}\text { Estrategias más } \\
\text { utilizadas/más valoradas }\end{array}$ & $\begin{array}{l}\text { Orientación (D: } \\
\text { Directiva, C: } \\
\text { Constructivista) }\end{array}$ & Hallazgos similares previos \\
\hline Pregunta & C & $\begin{array}{l}\text { Bocquillon et al. (2015); Correa-Molina } \\
\text { et al. (2014); Guerra (2009); Perrenoud } \\
\text { (2001); Ruffinelli (2018) }\end{array}$ \\
\hline Juicio/Sugerencia & D & Ruffinelli (2018) \\
\hline $\begin{array}{l}\text { Estructurar la reflexión por } \\
\text { dimensiones }\end{array}$ & C & Chung y Van Es (2014) \\
\hline Modelar & D & Russel (2012) \\
\hline Describir & D & Bocquillon et al. (2015) \\
\hline Estrategias menos utilizadas/bien valoradas & \\
\hline Análisis de casos & C & Cruickshank (1987); Perrenoud (2001) \\
\hline Simulaciones & C & Korthagen et al. (2001) \\
\hline Diarios reflexivos & C & Perrenoud (2001) \\
\hline $\begin{array}{l}\text { Filmación de clases y análisis } \\
\text { de prácticas }\end{array}$ & C & Cruickshank (1987); Perrenoud (2001) \\
\hline
\end{tabular}

Fuente: Elaboración propia.

\subsection{Sobre el mejoramiento de la tutoría}

Para el mejoramiento de la tutoría ambos actores coinciden en la necesidad de incrementar los tiempos destinados. Los tutores enfatizan la necesidad de profesionalizar esta actividad curricular y la demanda por vincularse más profundamente a la integralidad del proceso formativo, mientras que los practicantes abogan por la incorporación de otros actores relevantes a la tutoría: profesores guías, pares y estudiantes de aula, por velar que el tutor sea especialista en el ciclo educativo correspondiente y por la articulación de cursos cuyo diseño fue pensado de ese modo, pero que en la práctica se han disociado (Talleres de Reflexión y Práctica). 
En resumen, las proyecciones de los hallazgos hablan de la necesidad de realizar más investigación empírica que genere conocimiento acerca de cómo profesionalizar el rol del tutor, en el sentido que proponen Ball y Forzani (2011) de construir un núcleo común de saberes y habilidades para la enseñanza entre los tutores de futuros profesionales de la educación, derivados de la investigación, que propongan formas de vincular este rol y esta actividad curricular efectivamente al resto del proceso formativo (que hoy aparece en una perspectiva satelital desde la precaria vinculación del tutor con los programas hasta las definiciones de los objetivos y medios), y de avanzar al trabajo de coconstrucción colaborativa respecto de la estructura, función, estrategias e instrumentos de la tutoría, en un marco de consistencia, comunicación y tributo al proceso formativo mayor.

Los hallazgos muestran también la necesidad de incorporar nuevos actores al trabajo tutorial, fundamentalmente a los profesores guías, los pares y la voz de los propios estudiantes del practicante en la escuela. En particular refieren a incorporar al profesor guía en un rol formativo, trascendiendo al rol de un evaluador o calificador del desempeño del practicante desde la paradojal tensión de su visión personal como docente en ejercicio y las demandas de unas pautas evaluativas diseñadas unilateralmente por la universidad, en una lógica de demanda externa débilmente consensuada.

Esto significa avanzar hacia la constitución de la tríada formativa, cuestión que demanda: desarrollo y uso de hallazgos investigativos que orienten una efectiva co construcción reflexiva y colaborativa del espacio formativo con la escuela, develar formas en que puedan lograrse acuerdos en las comprensiones, criterios, objetivos y medios relativos a este proceso, resignificar roles, valorar el rol formativo del profesor guía y de la formación de tutores y guías. Todo, a partir de investigación empírica y situada que sea capaz de sostener el intencionamiento del desarrollo profesional de los tutores, reconfigurando el espacio formativo y sus prácticas pedagógicas. 


\section{Referencias bibliográficas}

Altet, M. (1996). Les compétences de l'enseignant-professionnel: entre savoirs, schèmes d'action et adaptation, le savoir analyser. En L. Paquay, M. Altet, E. Charlier, \& P. Perrenoud (Eds.). Former des enseignants professionnels (pp. 27-40). Bruxelles: De Boeck Université.

Ambrosetti, A. (2010). The Interconnectedness of the Roles of Mentorsand Mentees in Preservice Teacher Education Mentoring Relationships. Australian Journal of Teacher Education, 35, 42-55.

Ball, D., \& Forzani, F. (2011). Building a Common Core for Learning to Teach: And Connecting Professional Learning to Practice. American Educator, 35(2), 17-21, 38-39.

Bates, A., Ramírez, L., \& Drits, D. (2009). Connecting University Supervision and Critical Reflection: Mentoring and Modeling. Teacher Educator, 44(2), 90-112.

Blomberg, G., Sherin, M., Renkl, A., Glogger, I., \& Seidel, T. (2014). Understanding Video as a Tool for Teacher Education: Investigating Instructional Strategies to Promote Reflection. International Journal of the Learning Sciences, 42(3), 443-463.

Bocquillon, M., Dehon, A., \& Derobertmasure, A. (2015). Interventions du superviseur lors de séances de rétroaction visant le développement de la réflexivité: étude de la variabilité inter-superviseurs. Phronesis, 4(1), 14-27.

Butler, D. L., \& Winne, P. H. (1995). Feedback and Self-Regulated Learning: A Theoretical Synthesis. Review of Educational Research, 65(3), 245-281.

Chambers, F. C., \& Armour, K. M. (2011). Do as we do and not as we say: teacher educators supporting student teachers to learn on teaching practice. Sport Education and Society, 16(4), 527-544.

Chung, H., \& Van Es, E. (2014). Pre-service teachers' use of tools to systematically analyze teaching and learning. Teachers and Teaching, 20(2), 113-135.

Concha, S., Hernández, C., Del Río, F., Romo, F., \& Andrade, L. (2013). Reflexión pedagógica en base a casos y dominio de lenguaje académico en estudiantes de cuarto año de pedagogía en educación básica. Calidad en la educación, 38, 81-113.

Cornejo, J. (2014). Prácticas profesionales durante la formación inicial docente: análisis y optimización de sus aportes a los que aprenden y a los que enseñan a aprender "a enseñar". Estudios Pedagógicos, 40, 239-256. 
Correa-Molina, E. (2009). El supervisor de prácticas: recursos para una supervisión eficaz. Pensamiento Educativo, 44-45, 237-254.

Correa-Molina, E. (2011). La práctica docente: una oportunidad de desarrollo profesional. Perspectiva Educacional, 50(2), 77-95.

Correa-Molina, E., Chaubet, P., Collin, S., \& Gervais, C. (2014). Desafíos metodológicos para el estudio de la reflexión en contexto de formación docente. Estudios Pedagógicos, 40(1), 71-86.

Cruickshank, D. (1987). Reflective teaching: The preparation of students of teaching. Reston, VA: Association of Teacher Educators.

Dewey, J. (1989). Cómo pensamos. Nueva exposición de la relación entre pensamiento reflexivo y proceso educativo. Barcelona: Paidós.

Douglas, A. S., \& Ellis, V. (2011). Connecting does not necessarily mean learning: Course handbooks as mediating tools in school-university partnerships. Journal of Teacher Education, 62(5), 465-476.

Gaudin, C., \& Chaliès, S. (2012). L'utilisation de la vidéo dans la formation professionnelle des enseignants novices: revue de littérature et zones potentielles d'étude. Revue Française de Pédagogie, 178, 115-130.

Geber, H., \& Nyanjom, J. A. (2009). Mentor Development in Higher Education in Botswana: How Important Is Reflective Practice? South African Journal of Higher Education, 23(5), 894911.

Gelfuso, A., \& Dennis, D. (2014). Getting reflection off the page: The challenges of developing support structures for pre-service teacher reflection. Teaching and Teacher Education, 38, 1-11.

Gervais, G., \& Correa-Molina, E. (2004). Explicitación del saber de experiencia de los profesores en el contexto de las prácticas docentes. Un marco conceptual y metodológico. Íkala, 9(15), 141-167.

Guerra, P. (2009). Revisión de experiencia de reflexión en la formación inicial de docentes. Estudios pedagógicos, 35(2), 243-260.

Hattie, J., \& Timperley, H. (2007). The Power of Feedback. Review of Educational Research, 77(1), 81-112.

Hirmas, C. (2014). Conclusiones, tensiones y olvidos en la formación práctica docente. En I. Cortés, \& C. Hirmas (Eds.), Primer Seminario sobre Formación Práctica Docente: Vinculación entre el sistema universitario y el sistema escolar (pp. 8-21). Santiago de 
Chile: Organización de Estados Iberoamericanos para la Educación de la Ciencia y la Cultura.

Hirmas, C., \& Cortés, I. (2015). Estado del arte, Investigaciones sobre formación práctica en Chile. Tensiones y desafíos. Santiago de Chile: Organización de Estados Americanos para la Educación, la Ciencia y la Cultura.

Hudson, P. (2005). Identifying mentoring practices for developing effective primary science teaching. International Journal of Science Education, 27(14), 1723-1739.

Hudson, P., Usak, M., \& Savran-Gencer, A. (2009). Employing the five-factor mentoring instrument: analysing mentoring practices for teaching primary science. European Journal of Teacher Education, 32(1), 63-74.

Jaeger, E. (2013). Teacher Reflection: Supports, Barriers, and Results. Issues in Teacher Education, 22(1), 89-104.

Janssen, F., De Hullu, E., \& Tigelaar, D. (2008). Positive Experiences as Input for Reflection by Student Teachers. Teachers and Teaching: Theory and Practice, 14(2), 115-127.

Jones, M., \& Ryan, J. (2014). Learning in the practicum: engaging pre-service teachers in reflective practice in the online space. Asia-Pacific Journal of Teacher Education, 42(2), 132-146.

Korthagen, F., Kessels, J., Koster, B., Lagerwerf, B., \& Wubbels, T. (2001). Linking practice and theory: The pedagogy of realistic teacher education. Mahwah, NJ: Erlbaum.

Lawson, T., Çakmak, M., Gündüz, M., \& Busher, H. (2015). Research on teaching practicum - a systematic review. European Journal of Teacher Education, 38(3), 392-407.

Lincoln, Y. S., \& Guba, E. G. (1985). Naturalistic Inquiry. Newbury Park, CA: Sage.

Maes, M., Colognesi, A., \& Van Nieuwenhoven, A. (2018). «Accompagner/former» ou «évaluer/vérifier». Education \& Formation, 308, 95-106.

Meireu, P. (1998). Frankenstein educador. Barcelona: Laertes.

Moore-Russo, D., \& Wilsey, J. (2014). Delving into the meaning of productive reflection: A study of future teachers' reflections on representations of teaching. Teaching and Teacher Education, 37, 76-90.

Moscovici, S. (1984). The phenomenon of social representations. En R. Farr, \& S. Moscovici (Eds.), European Studies in Social Psychology (pp. 3-69). Cambridge: Cambridge University Press.

Perrenoud, P. (2001). Desarrollar la práctica reflexiva en el oficio de enseñar. Profesionalización y razón pedagógica. Barcelona: Grao. 
Puig-Cruells, C. (2004). El rol docente del tutor de prácticas y el acontecimiento al estudiante. Portularia, 4, 455-462.

Romero-Jeldres, M., \& Alcaíno-Opazo, V. (2014). Creencias y estilos de supervisión de profesores supervisores de prácticas: Resultados en una muestra exploratoria. Estudios Pedagógicos, 40(2), 321-340.

Rubenstein, E. D., \& Thoron, A. C. (2013). An Observational Analysis of Agricultural Education Faculty During On-Site Supervisory Visits with Pre-service Teachers. Journal of Agricultural Education, 54(4), 134-148.

Ruffinelli, A. (2018). Reflexión docente: oportunidades de desarrollo en la formación inicial. (Tesis doctoral). Recuperado desde https://repositorio.uc.cl/handle/11534/22001

Russell, T. (2012). Cambios paradigmáticos en la formación de profesores: Peligros, trampas y la promesa no cumplida del profesional reflexivo. Encounters on Education, 13, 71-91.

Sánchez, G., \& Jara, X. (2014). Los espacios de tutoría en práctica profesional y sus necesidades de fortalecimiento. Actualidades Investigativas en Educación, 14(2), 1-25.

Simpson, T., Hasting, W., \& Hill, B. (2007). "I knew that she was watching me": the professional benefits of mentoring. Teachers and Teaching: Theory and Practice, 13(5), 481-498.

Strauss, A., \& Corbin, J. (1998). Basics of qualitative research: Techniques and procedures for developing grounded theory. Thousand Oaks, CA: Sage.

Wagner, W. (1994). Speaking is acting is representation-comments on the reply by A. Echebarría. Papers on Social Representations, 3, 201-206. 